\title{
The efficacy of TACE; how can automated feeder software help?
}

\author{
Hassan Abdelsalam ${ }^{*}$ (D), Doaa M. Emara ${ }^{1}$ and Ehab M. Hassouna ${ }^{2}$
}

\begin{abstract}
Background: This is a prospective study of 44 patients having 57 hepatocellular carcinoma (HCC) lesions indicated for transarterial chemoembolization (TACE) and a control group of 41 patients having 55 HCC lesions. TACE in the study group was performed on an angiography machine with an installed automated feeder detection (AFD) software (EmboGuide; Philips Healthcare, Best, The Netherlands) and in the control group was performed on a similar angiography machine (ALLURA XPER FD 20, Philips, Holland), but lacking the AFD software. The aim is to evaluate the clinical utilization of cone-beam CT (CBCT) and (AFD) software in accurate detection of (HCC) arterial feeders, the effect of (AFD) software utilization on the outcome of (TACE) and its utilization effect on fluoroscopy time and radiation dose to the patient during the (TACE) procedure.

Results: The highest percentage of agreement was between the number of arterial feeders detected by EmboGuide and the actual number of feeders detect during embolization reaching $91.2 \%$ with $p$ value $<0.001$. The residual non-intended non-embolized areas among the study group at 1-month follow-up were found in 2 out of 57 lesions compared to 9 out of 55 lesions in the control group. The average dose-area product (DAP) among the study group was less than that of the control group.
\end{abstract}

Conclusion: CBCT using AFD software provides more information about tumor feeders with consequent more efficient targeted embolization, higher success rate of TACE and less patient exposure to radiation.

Keywords: TACE, EmboGuide, HCC feeders

\section{Background}

Hepatocellular carcinoma (HCC) is the most common malignant tumor on top of cirrhotic liver, and it showed increased incidence over the last two decades and is considered the fifth most common cancer and the third leading cause of cancer deaths [1, 2]. Trans-arterial chemoembolization (TACE) is considered the treatment of choice for inoperable HCC cases [3-5]. Accurate detection of the arterial feeder of $\mathrm{HCC}$ is an important key for success of TACE and hence decreases the rate of recurrence after embolization [6].

\footnotetext{
*Correspondence: Hassan_abd_elsalam@yahoo.com

1 Radiology Department, Faculty of Medicine, Alexandria University,

Alexandria, Egypt

Full list of author information is available at the end of the article
}

Conventional digital subtraction angiography (DSA) in different angles for detection of the arterial feeder is the routine method used in TACE; however, it is time-consuming with increased exposure to radiation [6]. Conebeam CT (CBCT) has been used recently not only for detection of tumors but also to detect the arterial feeder accurately using CBCT angiography with $3 \mathrm{D}$ vessel tracking system with a single acquisition of C-arm CT $[3,4,7]$. A new software has been recently developed by different companies known as automated feeder detection (AFD) software that can identify the tumor feeders automatically on the workstation as a color-coded image $[6,8]$.

The primary aim of this study was to evaluate the clinical utilization of CB-CT and automated feeder detection (AFD) software in accurate detection of HCC feeding arteries and the effect on the outcome of transarterial chemoembolization (TACE). A secondary aim was to 
assess the effect of utilization of (AFD) software on the fluoroscopy time of the procedure of TACE and the radiation dose to the patient during the procedure.

\section{Methods}

\section{Patients}

This is a prospective study of patients referred to two interventional radiology units presenting with hepatocellular carcinoma (HCC) indicated for transarterial chemoembolization (TACE). Patients with infiltrative HCC, vascular invasive lesions and lesions with extrahepatic arterial supply were excluded as well lesions with recurrence after initial loco-regional management.

The study group consists of 44 patients having $57 \mathrm{HCC}$ lesions and a control group of 41 patients with 55 HCC lesions. Patients with multiple lesions were embolized at the same session. This is a non-randomized study; the patients were referred to two different institutions in the same city; however, both groups were treated by the same interventional radiologist.

The study protocol was approved by the ethical committee of our university hospital, and an informed consent was obtained from each patient included in the study.

\section{Procedure}

All TACE procedures were done on angiography machine (ALLURA XPER FD 20, Philips, Holland), the study group was done on a machine with installed automated feeder detection (AFD) software (EmboGuide; Philips Healthcare, Best, The Netherlands), and the control group was done on a similar angiography machine, but lacking the AFD software. All TACE procedures were carried out by the same interventional radiologist who has more than 15-year experience in performing TACE.

The TACE procedure was done in a standard technique through a femoral puncture. In total, $30 \mathrm{ml}$ of non-diluted contrast (Ultravist 300, Bayer Inc., Ontario, Canada) was injected via a pump at a rate of $3 \mathrm{ml} / \mathrm{s}$ into a 5-French catheter placed in the common or proper hepatic artery and a rotational hepatic arteriography was obtained, the data were processed automatically by the machine producing the $\mathrm{CBCT}$ images. The $\mathrm{CBCT}$ images were reviewed by two experienced interventional radiologists (observer A) and (observer B) separately to detect and document the number of arterial feeders for each lesion. The AFD software (EmboGuide) was then applied by placing circular regions-of-interest (ROIs) for each lesion, and the tip of the catheter was marked on 2D MPR images. The software marked each arterial feeder in a different color. The number of feeders for each lesion was documented. A microcatheter was then introduced superselectively for each lesion. Contrast was injected through the microcatheter to confirm the catheter tip position and to start chemoembolization. The number of feeders when the target lesion enhanced was documented. Digital subtraction angiography runs were done when needed by the radiologist. Another cone-beam CT is usually performed at the end of the chemoembolization session to assure that there are no defects in embolization and that the lesion is completely embolized. The radiation dose and fluoroscopy time for each patient are recorded by the machine and documented. The same procedure of chemoembolization including the conebeam CT was done for the control group. The only difference is that the angiography machine lacks the AFD software.

All patients had a follow-up triphasic liver CT to assess tumor embolization and presence of residual non-embolized parts of the lesion. Residual lesion was confirmed on CT by the presence of an abnormal early enhancement in the arterial phase; thus, the lesion is reported on $\mathrm{CT}$ as LI-RADS treatment response (LRTR) viable.

\section{Statistical analysis}

Data were fed to the computer and analyzed using IBM SPSS software package version 20.0. (Armonk, NY: IBM Corp). Comparisons between different categorical variables were assessed using Chi-square test (Fisher or Monte Carlo). Marginal homogeneity test was assessed for comparison between two periods for normally distributed quantitative variables. Student's t-test was used to compare two groups for normally distributed quantitative variables. Mann-Whitney test was used to compare between two groups for not normally distributed quantitative variables. Significance of the obtained results was judged at the $5 \%$ level.

\section{Results}

The current study included two groups: our cases (A) and the control (B);

\section{Cases (Group A)}

Included 44 patients with 57 lesions. 27 males and 17 females, and the age ranged from 42 to 71 years.

According to their Child Pugh scores, 25 patients were class A and 19 were class B.

The size of the lesions ranged from 1.9 to $4.8 \mathrm{~cm}$ with mean size was $3.2 \mathrm{~cm}$.

Thirty-three patients had single lesions, while nine had 2 lesions each, and two had 3 lesions each.

\section{Control (Group B)}

Included 41 patients with 55 lesions. 26 males and 15 females, and the age ranged from 39 to 72 years. 
According to their Child Pugh scores, 22 patients were class A and 19 were class B.

The size of the lesions ranged from 1.6 to $4.7 \mathrm{~cm}$ with mean size was $3 \mathrm{~cm}$.

Twenty-seven patients had single lesions, while 14 had more two lesions each.

Comparison of the demographic data, Child Pugh classification and lesion characteristics between the study and control groups revealed no statistically significant difference between the two groups (Table 1).

\section{Number of arterial feeders}

We compared the number of feeders among the studied cases detected by observer A, observer B as well as the feeders detected by using EmboGuide software and the actual number of feeders detected during angiography. There was a significant difference in the number of feeders reported by observers $\mathrm{A}$ and $\mathrm{B}$ compared to the actual feeders $(p=0.002$ and $p=0.004)$, respectively. Meanwhile, there was no significant difference between the number of feeders detected by EmboGuide and the actual feeders $(p=0.180)$ (Table 2).

Percentage of agreement between observer $\mathrm{A}$ and the actual number of feeders was $54.4 \%$ with $p$ value $<0.001$. Percentage of agreement between observer $B$ and the actual number of feeders was $43.8 \%$ with $p$ value $<0.001$, while the highest percent of agreement was between the number of arterial feeders detected by EmboGuide and the actual number of feeders reaching $91.2 \%$ with $p$ value $<0.001$ (Table 3 ).
Table 2 Comparison between actual with observer A \& observer B and EmboGuide $(n=57)$

\begin{tabular}{lllll}
\hline \multicolumn{5}{l}{ Number of arterial feeders detected } \\
\cline { 2 - 5 } & Observer A & Observer B & EmboGuide & Actual \\
\hline 1 & $15(26.3 \%)$ & $16(28.1 \%)$ & $12(21.1 \%)$ & $12(21.1 \%)$ \\
2 & $23(40.4 \%)$ & $24(42.1 \%)$ & $17(29.8 \%)$ & $20(35.1 \%)$ \\
3 & $16(28.1 \%)$ & $11(19.3 \%)$ & $17(29.8 \%)$ & $14(24.6 \%)$ \\
4 & $3(5.3 \%)$ & $6(10.5 \%)$ & $11(19.3 \%)$ & $11(19.3 \%)$ \\
$p$ & $0.002^{*}$ & $0.004^{*}$ & 0.180 & \\
\hline
\end{tabular}

$p: p$ value for marginal homogeneity test for comparison between actual with observer A \& observer B and EmboGuide

${ }^{*}$ : Statistically significant at $p \leq 0.05$

We compared the number of feeders among the studied cases by observer A, observer B as well as the feeders detected by using the EmboGuide software (Table 4).

There was agreement between observer A and EmboGuide of about $51 \%$ with $p$ value $<0.001$, while percent of agreement between EmboGuide and observer B was $45.6 \%$ with $p$ value $<0.001$ (Table 5).

Complete devascularization was achieved in all 57 lesions (100\%) of group A and 55 lesions (100\%) of group $\mathrm{B}$ during the TACE session.

Figure 1 shows the steps of $\mathrm{CBCT}$, EmboGuide and TACE in one of our study cases.

Table 1 Comparison between the two studied groups according to different parameters

\begin{tabular}{|c|c|c|c|c|}
\hline & Cases $(n=44)$ & Control $(n=41)$ & Test of Sig. & $p$ \\
\hline \multicolumn{5}{|l|}{ Sex } \\
\hline Male & $27(61.4 \%)$ & $26(63.4 \%)$ & $x^{2}=0.038$ & 0.845 \\
\hline Female & $17(38.6 \%)$ & $15(36.6 \%)$ & & \\
\hline \multicolumn{5}{|l|}{ Age (years) } \\
\hline Mean $\pm S D$ & $60.1 \pm 8.3$ & $59.4 \pm 8.3$ & $t=0.375$ & 0.708 \\
\hline Median (Min.-Max.) & $60.5(42-71)$ & $60.0(39-72)$ & & \\
\hline \multicolumn{5}{|l|}{ Child Pugh class } \\
\hline A & $25(56.8 \%)$ & $22(53.7 \%)$ & $x^{2}=0.086$ & 0.770 \\
\hline B & $19(43.2 \%)$ & $19(46.3 \%)$ & & \\
\hline Lesion size $(\mathrm{cm})$ & $(n=57)$ & $(n=55)$ & & \\
\hline Mean $\pm S D$ & $3.2 \pm 0.9$ & $3.0 \pm 0.7$ & $t=1.293$ & 0.199 \\
\hline Median (Min.-Max.) & $2.9(1.9-4.8)$ & $2.9(1.6-4.7)$ & & \\
\hline Number of lesions & $(n=57)$ & $(n=55)$ & & \\
\hline 1 & $33(75.0 \%)$ & $27(65.9 \%)$ & $x^{2}=3.190$ & ${ }^{M C} p=0.156$ \\
\hline 2 & $9(20.5 \%)$ & $14(34.1 \%)$ & & \\
\hline 3 & $2(4.5 \%)$ & $0(0.0 \%)$ & & \\
\hline
\end{tabular}


Table 3 Percent of agreement between actual with observer A \& observer B and EmboGuide $(n=57)$

\begin{tabular}{|c|c|c|c|c|c|c|}
\hline & \multicolumn{4}{|l|}{ Actual } & \multirow[t]{2}{*}{$\%$ of Agreement } & \multirow[t]{2}{*}{$p$} \\
\hline & $1(n=12)$ & $2(n=20)$ & $3(n=14)$ & $4(n=11)$ & & \\
\hline \multicolumn{7}{|c|}{ Observer A } \\
\hline 1 & $12(100 \%)$ & $2(10 \%)$ & $1(7.1 \%)$ & $0(0 \%)$ & \multirow[t]{4}{*}{54.4} & \multirow[t]{4}{*}{$<0.001^{*}$} \\
\hline 2 & $0(0 \%)$ & $13(65.0 \%)$ & 10 (71.4\%) & $0(0 \%)$ & & \\
\hline 3 & $0(0 \%)$ & $5(25.0 \%)$ & $3(21.4 \%)$ & $8(72.7 \%)$ & & \\
\hline 4 & $0(0 \%)$ & $0(0 \%)$ & $0(0 \%)$ & $3(27.3 \%)$ & & \\
\hline \multicolumn{7}{|c|}{ Observer B } \\
\hline 1 & $9(75.0 \%)$ & $7(35.0 \%)$ & 0 (0.0\%) & $0(0.0 \%)$ & \multirow[t]{4}{*}{43.8} & \multirow[t]{4}{*}{$<0.001^{*}$} \\
\hline 2 & $2(16.7 \%)$ & $9(45.0 \%)$ & 13 (92.9\%) & $0(0.0 \%)$ & & \\
\hline 3 & $1(8.3 \%)$ & $4(20.0 \%)$ & $1(7.1 \%)$ & $5(45.5 \%)$ & & \\
\hline 4 & $0(0.0 \%)$ & $0(0.0 \%)$ & $0(0.0 \%)$ & $6(54.5 \%)$ & & \\
\hline \multicolumn{7}{|c|}{ EmboGuide } \\
\hline 1 & $12(100 \%)$ & $0(0 \%)$ & $0(0 \%)$ & $0(0 \%)$ & \multirow[t]{4}{*}{91.2} & \multirow[t]{4}{*}{$<0.001^{*}$} \\
\hline 2 & $0(0 \%)$ & $16(80 \%)$ & $1(7.1 \%)$ & $0(0 \%)$ & & \\
\hline 3 & $0(0 \%)$ & 4 (20\%) & 13 (92.9\%) & $0(0 \%)$ & & \\
\hline 4 & $0(0 \%)$ & $0(0 \%)$ & $0(0 \%)$ & 11 (100\%) & & \\
\hline
\end{tabular}

p: $p$ value for Chi-square test for association between actual with observer A \& observer B and EmboGuide

${ }^{*}$ : Statistically significant at $p \leq 0.05$

Table 4 Comparison between EmboGuide with observer A and observer $\mathrm{B}(n=57)$

\begin{tabular}{llll}
\hline & \multicolumn{3}{l}{ Number of arterial feeders detected } \\
\cline { 2 - 4 } & Observer A & Observer B & EmboGuide \\
\hline 1 & $15(26.3 \%)$ & $16(28.1 \%)$ & $12(21.1 \%)$ \\
2 & $23(40.4 \%)$ & $24(42.1 \%)$ & $17(29.8 \%)$ \\
4 & $16(28.1 \%)$ & $11(19.3 \%)$ & $17(29.8 \%)$ \\
$p$ & $3(5.3 \%)$ & $6(10.5 \%)$ & $11(19.3 \%)$ \\
\hline
\end{tabular}

$\mathrm{p}_{2}: p$ value for marginal homogeneity test for comparison between EmboGuide with observer $A$ and observer $B$

*: Statistically significant at $p \leq 0.05$

\section{Residual tumor activity in both groups at one-month follow-up CT}

Residual non-intended non-embolized areas among group $\mathrm{A}$ in the machine with EmboGuide software at the 1-month follow-up are 2 out of 57 lesions while residual non-intended non-embolized areas among group B in the similar machine without EmboGuide software in the 1-month follow-up are 9 out of 55 lesions in 41 patients (Table 6).

\section{Dose-area product (DAP) for both groups}

DAP is used for estimating stochastic risk to patients from exposure to radiation.

Table 5 Percent of agreement between EmboGuide with observer A and observer B $(n=57)$

\begin{tabular}{|c|c|c|c|c|c|c|}
\hline & \multicolumn{4}{|c|}{ EmboGuide } & \multirow[t]{2}{*}{$\%$ of Agreement } & \multirow[t]{2}{*}{$p$} \\
\hline & $1(n=12)$ & $2(n=17)$ & $3(n=17)$ & $4(n=11)$ & & \\
\hline \multicolumn{7}{|c|}{ Observer A } \\
\hline 1 & $12(100 \%)$ & $2(11.8 \%)$ & $1(5.9 \%)$ & $0(0 \%)$ & \multirow[t]{4}{*}{45.6} & \multirow[t]{4}{*}{$<0.001^{*}$} \\
\hline 2 & $0(0 \%)$ & $9(52.9 \%)$ & $14(82.4 \%)$ & $0(0 \%)$ & & \\
\hline 3 & $0(0 \%)$ & $6(35.3 \%)$ & $2(11.8 \%)$ & $8(72.7 \%)$ & & \\
\hline 4 & $0(0 \%)$ & $0(0 \%)$ & $0(0 \%)$ & $3(27.3 \%)$ & & \\
\hline \multicolumn{7}{|c|}{ Observer B } \\
\hline 1 & $9(75.0 \%)$ & $5(29.4 \%)$ & $2(11.8 \%)$ & $0(0.0 \%)$ & \multirow[t]{4}{*}{49.1} & \multirow[t]{4}{*}{$<0.001^{*}$} \\
\hline 2 & $2(16.7 \%)$ & 10 (58.8\%) & $12(70.6 \%)$ & $0(0.0 \%)$ & & \\
\hline 3 & $1(8.3 \%)$ & $2(11.8 \%)$ & $3(17.6 \%)$ & $5(45.5 \%)$ & & \\
\hline 4 & $0(0.0 \%)$ & $0(0.0 \%)$ & $0(0.0 \%)$ & $6(54.5 \%)$ & & \\
\hline
\end{tabular}

p: $p$ value for Chi-square test for association between EmboGuide with observer $A$ and observer $B$

${ }^{*}$ : Statistically significant at $p \leq 0.05$ 

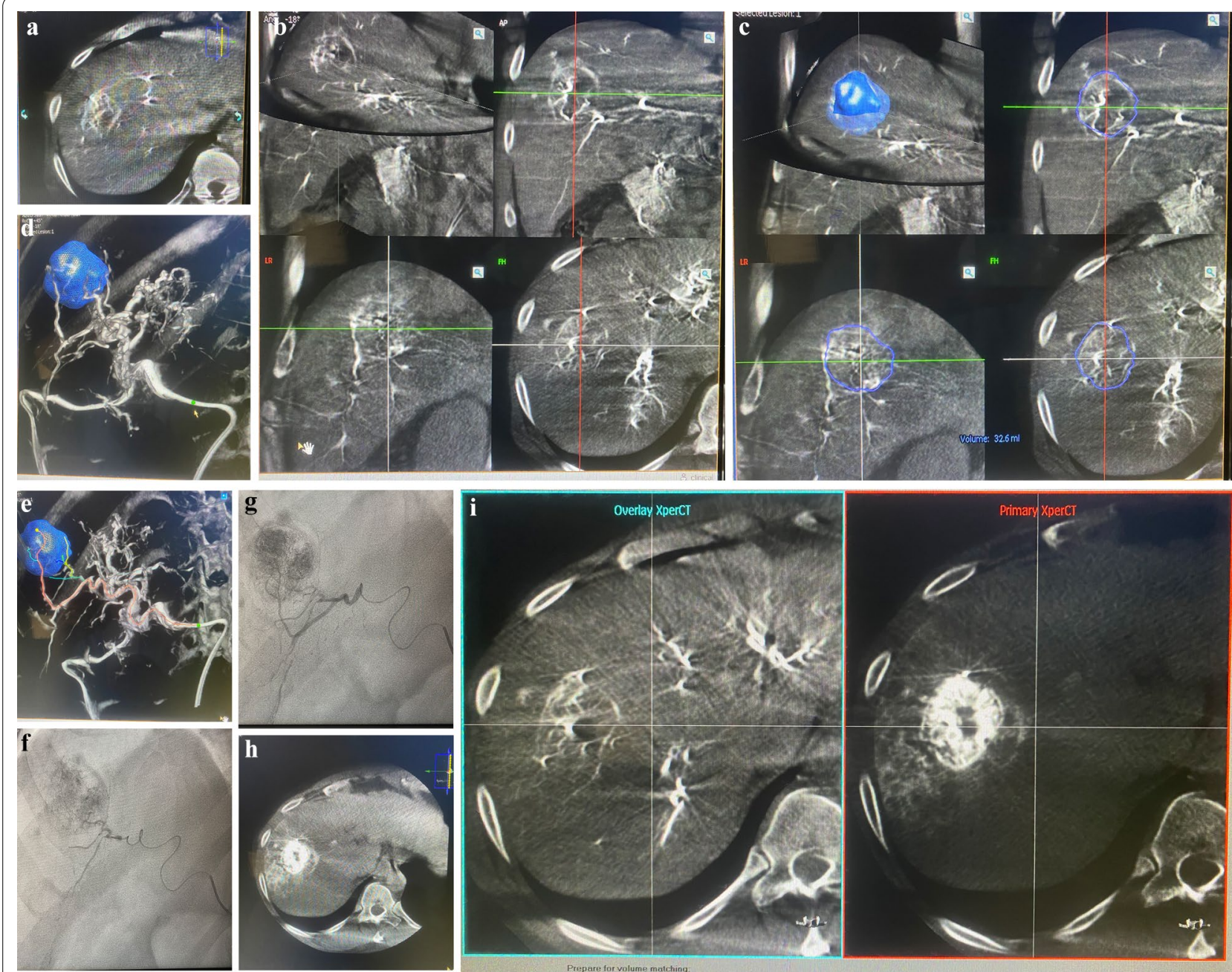

Fig. 1 The steps of CBCT, EmboGuide and TACE in one of our cases. a Cone-beam CT showing enhancing HCC lesion in segment VIII, $\mathbf{b}$ multiplanar image centered on the lesion. $\mathbf{c}$ Placing circular regions-of-interest (ROIs) for the lesion. $\mathbf{d}$ Placing a green dot at the tip of the catheter (arrow). e The software marked two arterial feeders colored in green and red. The green feeder gives a main branch (yellow) which gives smaller branches at the lesion site. The red feeder gives a small branch at the lesion site. f Fluoroscopic image with the tip of the microcatheter at the tip of the first feeder (marked green in the previous image) with injection of a mixture of Lipiodol and chemotherapy. $\mathbf{g}$ Fluoroscopic image showing the mixture of Lipiodol, and chemotherapy being injected in the second feeder (marked red in the previous image). $\mathbf{h}$ Completion CBCT showing the HCC completely embolized. i Comparison done by the machine software between the pre- and post-embolization CBCT to assure complete embolization of the lesion

Table 6 Comparison between the two studied groups according to residual lesion

\begin{tabular}{lcclc}
\hline & Cases $(\boldsymbol{n}=\mathbf{5 7})$ & Control $(\boldsymbol{n}=\mathbf{5 5})$ & $\boldsymbol{X}^{\mathbf{2}}$ & ${ }^{\mathrm{FE}_{\boldsymbol{p}}}$ \\
\hline No residual & $55(96.5 \%)$ & $46(83.6 \%)$ & $5.222^{*}$ & $0.028^{*}$ \\
Residual & $2(3.5 \%)$ & $9(16.4)$ & & \\
\hline
\end{tabular}

$x^{2}$ : Chi-square test, FE: Fisher exact

$p: p$ value for comparing between the two studied groups

${ }^{*}$ : Statistically significant at $p \leq 0.05$
The average DAPs for the 2 groups are as follows:

The average cone-beam CT DAP is 12,268 per CT.

The average digital subtraction angiographic run DAP is $62,379.7$ per run.

The average fluoroscopy DAP is $7316 \mathrm{mGycm}^{2}$ per min.

\section{In the study group (EmboGuide group)}

The average DAP (dose-area product) per patient is $128,829.3$ mGycm $^{2}$.

The average number of cone-beam CTs per patient is $1.8 \mathrm{CT}$. 
The average number of digital subtraction angiographic runs per patient is 0.6 run.

The average fluoroscopy time per patient is $9 \min 14 \mathrm{~s}$.

\section{Control group}

The average DAP per patient is 293,504.4 $\mathrm{mGycm}^{2}$.

The average number of cone-beam CT per patient is $1.7 \mathrm{CT}$.

The average number of digital subtraction angiographic run per patient is 2.4 run.

The average fluoroscopy time per patient is $16 \mathrm{~min}$, $47 \mathrm{~s}$.

We should take in consideration that the operator had to compensate for the absence of (EmboGuide) with more angiographic runs to make sure he is embolizing the target feeder(s) this has resulted in increased DAP to the patient as the DAP of the run is more than the DAP of the cone-beam CT. This also had an impact on fluoroscopy time, where the time has significantly increased in group B compared to group A.

There was a statistically significant difference among the two studied groups regarding the total DAP $\mathrm{mGycm}^{2}$ with $p$ value $<0.001$ and fluoroscopy time with $p$ value 0.002 (Table 7).

\section{Discussion}

Superselective TACE for HCC is important to increase the accuracy of embolization, decreasing the rate of recurrence and less harmful to the surrounding normal liver parenchyma [9]. Recent advances in imaging techniques help in diagnosis of HCC lesions even the small one; however, the tumor feeder detection in these cases with $2 \mathrm{D}$ angiography is usually difficult which will affect embolization of the lesion, the success rate, the prognosis and rate of recurrence [10]. Shiro Miyayama [11] mentioned that automated tumor feeder software is a recent technique, which is not widely available, that can be used for detection of tumor feeders accurately for superselective embolization with high success rate. Fortunately, we have used this novel software in the current study.
To our knowledge, there is paucity of the literature studying the role of AFD in accurate diagnosis of arterial feeders, prognosis of TACE and association with total patient radiation dose and fluoroscopy time; from this point we aimed to study all these factors collectively in our cases. By using the AFD software we detected the arterial feeders of HCC accurately which led to decreased rate of recurrence, using less radiation dose to the patient in a shorter fluoroscopic time; in other words, we could reach the maximum response with the least harm for HCC patients.

Our results showed the high percentage of agreement between the AFD software and the actual number feeders (91.2\%) compared to the percentage of our observers (54.4\% and $43.8 \%$, respectively). Not only that, but also the rate of recurrence among the studied cases (3.5\%) was less than that of control group (16.4\%) with statistically significant difference. Jin et al. [6] found that the sensitivity of the automated software in feeder detection was significantly higher than that of manual assessment using DSA ( $87.7 \%$ vs. $71.8 \%, P<0.001)$ with consequently proper embolization and decreased rate of recurrence. Melanie et al. [12] and Yasunori et al. [7] found high sensitivity of using AFD software during TACE.

In our study group, the average number of cone-beam CTs per patient is $(1.8 \mathrm{CT})$ which was not different than the control group (1.7 CT), while the average number of digital subtraction angiographic runs per patient was less in the study group ( 0.6 run) compared to ( 2.4 run) in the control group. These results compared well to Iawazawa et al. [8] who found that the overall angiography acquisition reduced from 6.6 times to 4.6 times with the assistance of software $(p<0.001)$. The number of $\mathrm{C}$-arm $\mathrm{CT}$ acquisitions per session did not differ between the groups.

Jin et al. [6] suggested-but have not studied-that using automated feeder software can reduce the number of acquisitions, fluoroscopy time and hence the total radiation dose as well as the total dose of contrast used. In the current study, we studied these items

Table 7 Comparison between the two studied groups according to total DAP and fluoroscopy

\begin{tabular}{llll}
\hline & Cases $(\boldsymbol{n}=\mathbf{4 4})$ & Control $(\boldsymbol{n}=\mathbf{4 1})$ & $\boldsymbol{U}$ \\
\hline Total DAP mGycm ${ }^{2}$ & & & $\boldsymbol{p}$ \\
Mean \pm SD & $128,829.2 \pm 65,668.9$ & $293,504.6 \pm 131,823.1$ & $204.0^{*}$ \\
Median (Min.-Max.) & $105,458(64,794-294,516)$ & $287,929(110,408-636,094)$ & $<0.001^{*}$ \\
Fluoroscopy time in min & & & \\
Mean \pm SD & $9.24 \pm 2.23$ & $16.74 \pm 10.98$ & $546.0^{*}$ \\
Median (Min.-Max.) & $9.17(4.48-13.48)$ & $14.10(4.20-44.30)$ & $0.002^{*}$ \\
\hline
\end{tabular}

U: Mann-Whitney test, p: $p$ value for comparing between the two studied groups

${ }^{*}$ : Statistically significant at $p \leq 0.05$ 
and found significant reduction in total fluoroscopic time in the study group compared to the control group $(9.24 \pm 2.23 \mathrm{~min})$ and $(16.74 \pm 10.98 \mathrm{~min})$, respectively. The average DAP was $128,829.2 \pm 65,668.9 \mathrm{mGycm}^{2}$ in our study group which is significantly lower than that of the control group which was $293,504.6 \pm 131,823.1$ $\mathrm{mGycm}^{2}$; we believe this is due to less number angiography runs needed with the usage of AFD software. This was in agreement with Vania et al. [3] who mentioned the efficacy of using CBCT that gives 3D volumetric data with decreasing the cumulative effect of radiation as compared to routine DSA. They concluded that this technique is essential to be used by intervention radiologist during TACE.

In the current study residual tumor, in one-month follow-up, was detected only in two out of 57 lesions in group A, while that in group B not using AFD was higher; nine out of 55 lesions with statistically significant difference comparing the two groups, these data match the findings. Miyayama et al. [10] confirmed presence of higher rate of complete and even partial response in their cases with less rate of recurrence.

\section{Conclusion}

CBCT using AFD software is a recent technique which provides more information than 2D standard angiography about the tumor feeders, higher success rate of TACE with greater confidence that can be assessed during the procedure, not only that but also the total exposure to radiation when using CBCT is less than that of $2 \mathrm{D}$ angiography. So we can improve the efficacy of TACE by using AFD software.

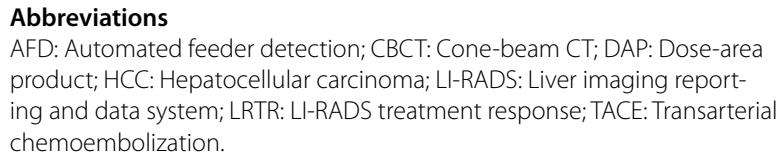
product; HCC: Hepatocellular carcinoma; LI-RADS: Liver imaging reporting and data system; LRTR: LI-RADS treatment response; TACE: Transarterial chemoembolization.

\begin{abstract}
Authors' contributions
HA, Conception and design of the work, Acquisition, analysis, and interpretation of data; Drafted the work and substantively revised it. DME, Conception and design of the work, Acquisition, analysis, and interpretation of data; Drafted the work and substantively revised it. EMH, Acquisition of data. All authors have approved the submitted version (and any substantially modified version that involves the author's contribution to the study); All authors have agreed both to be personally accountable for the author's own contributions and to ensure that questions related to the accuracy or integrity of any part of the work, even ones in which the author was not personally involved, are appropriately investigated, resolved, and the resolution documented in the literature.
\end{abstract}

\section{Funding}

There was no funding for this study.

\section{Availability of data and materials}

The datasets used and/or analyzed during the current study are available from the corresponding author on reasonable request.

\section{Declarations}

\section{Ethics approval and consent to participate}

All procedures performed in studies involving human participants were in accordance with the ethical standards of the institutional research committee (Ethics committee, Faculty of Medicine, Alexandria University). Approval Serial number 0304376, Committee IRB number 00012098. Informed consent was obtained from all individual participants included in the study. This article does not contain patient data.

\section{Consent for publication}

Not applicable.

\section{Competing interests}

All authors declare that they have no conflict of interest. All authors certify that they have no affiliations with or involvement in any organization or entity with any financial interest or non-financial interest in the subject matter or materials discussed in this manuscript.

\section{Author details}

${ }^{1}$ Radiology Department, Faculty of Medicine, Alexandria University, Alexandria, Egypt. ${ }^{2}$ Internal Medicine Department, Faculty of Medicine, Alexandria University, Alexandria, Egypt.

Received: 28 November 2021 Accepted: 5 February 2022

Published online: 15 February 2022

\section{References}

1. Petrick JL, Kelly SP, Altekruse SF, McGlynn KA, Rosenberg PS (2016) Future of hepatocellular carcinoma incidence in the United States Forecast through 2030. J Clin Oncol 34(15):1787-1794

2. Wang $X$, Yarmohammadi $H$, Cao G, Ji X, Hu J, Yarmohammadi $H$, Chen H, Zhu X, Yang R, Solomon SB (2017) Dual phase cone-beam computed tomography in detecting $<3 \mathrm{~cm}$ hepatocellular carcinomas during transarterial chemoembolization. J Can Res Ther 13:38-43

3. Tacher V, Radaelli A, Lin M, Geschwind JF (2015) How I do it: conebeam CT during transarterial chemoembolization for liver cancer. Radiology 274:320-334

4. Miyayama S, Yamashiro M, Nagai K, Tohyama J, Kawamura K, Yoshida M, Sakuragawa N (2016) Efficacy of automated tumor-feeder detection software using cone-beam computed tomography technology in transarterial embolization through extrahepatic collateral vessels for malignant hepatic tumors. Hepatol Res 46:166-173

5. Lee IJ, Chung JW (2019) Conventional chemoembolization for hepatocellular carcinoma: role of cone-beam computed tomography guidance. J Liver Cancer 19(1):19-29

6. Iwazawa J, Ohue S, Hashimoto N, Muramoto O, Mitani T (2013) Clinical utility and limitations of tumor-feeder detection software for liver cancer embolization. Eur J Radiol 82(10):1665-1671

7. Minami Y, Murakami T, Kitano M, Sakurai T, Nishida N, Kudo M (2015) Cone-beam CT angiography for hepatocellular carcinoma: current status. Dig Dis 33:759-764

8. Iwazawa J, Ohue S, Hashimoto N, and Mitani T. Comparison of the number of image acquisitions and procedural time required for transarterial chemoembolization of hepatocellular carcinoma with and without tumor-feeder detection software. Radiol Res Pract. 2013;Article ID 580839

9. Pung L, Ahmad M, Mueller K, Rosenberg J, Stave C, Hwang GL, Shah R, Kothary N (2017) The role of cone-beam CT in transcatheter arterial chemoembolization for hepatocellular carcinoma: a systematic review and meta-analysis. JVIR 28(3):334-341

10. Miyayama S, Yamashiro M, Ikuno M, Okumura K, Yoshida M (2014) Ultraselective transcatheter arterial chemoembolization for small hepatocellular carcinoma guided by automated tumor-feeders detection software: technical success and short-term tumor response. Abdom Imaging 39(3):645-656

11. Miyayama S (2020) Treatment Strategy of Transarterial Chemoembolization for Hepatocellular Carcinoma. Appl Sci 10(20):7337 
12. Chiaradia M, Izamis ML, Radaelli A, Prevoo W, Maleux G, Schlachter T, Mayer J, Luciani A, Kobeiter H, Tacher V (2018) Sensitivity and reproducibility of automated feeding artery detection software during transarterial chemoembolization of hepatocellular carcinoma. JVIR 29(3):425-431

\section{Publisher's Note}

Springer Nature remains neutral with regard to jurisdictional claims in published maps and institutional affiliations.

\section{Submit your manuscript to a SpringerOpen ${ }^{\circ}$ journal and benefit from:}

- Convenient online submission

- Rigorous peer review

- Open access: articles freely available online

- High visibility within the field

- Retaining the copyright to your article

Submit your next manuscript at $\boldsymbol{\nabla}$ springeropen.com 